\title{
Maternal immune conditions are increased in males with autism spectrum disorders and are associated with behavioural and emotional but not cognitive co-morbidity
}

\author{
Shrujna Patel ${ }^{1}$, Russell C. Dale ${ }^{2}$, Destanie Rose ${ }^{3}$, Brianna Heath ${ }^{4}$, Christine W. Nordahl ${ }^{4}$, Sally Rogers ${ }^{4}$, \\ Adam J. Guastella ${ }^{1}$ and Paul Ashwood (iD ${ }^{3}$
}

\begin{abstract}
Epidemiological and animal research shows that maternal immune activation increases the risk of autism spectrum disorders (ASD) in offspring. Emerging evidence suggests that maternal immune conditions may play a role in the phenotypic expression of neurodevelopmental difficulties in children with ASD and this may be moderated by offspring sex. This study aimed to investigate whether maternal immune conditions were associated with increased severity of adverse neurodevelopmental outcomes in children with ASD. Maternal immune conditions were examined as predictors of ASD severity, behavioural and emotional well-being, and cognitive functioning in a cohort of 363 children with ASD ( $n=363 ; 252$ males, 111 females; median age 3.07 [interquartile range 2.64-3.36 years]). We also explored whether these outcomes varied between male and female children. Results showed that maternal asthma was the most common immune condition reported in mothers of children with ASD. A history of maternal immune conditions $(p=0.009)$ was more common in male children with ASD, compared to female children. Maternal immune conditions were associated with increased behavioural and emotional problems in male and female children. By contrast, maternal immune conditions were not associated with decreased cognitive function. The findings demonstrate that MIA may influence the expression of symptoms in children with ASD and outcomes may vary between males and females.
\end{abstract}

\section{Introduction}

Autism spectrum disorder (ASD) is a pervasive neurodevelopmental disorder that typically appears in childhood. ASD affects 1 in 54 people in the United States ${ }^{1}$ and this rate has been increasing over time. ASD is characterized by impairments in social communication and interaction, as well as the presence of repetitive and restricted behaviours or interests ${ }^{2}$. The clinical

\footnotetext{
Correspondence: Paul Ashwood (pashwood@ucdavis.edu)

${ }^{1}$ Autism Clinic for Translational Research, Brain and Mind Centre, Children's

Hospital Westmead Clinical School, Faculty of Medicine and Health, University of Sydney, Camperdown, NSW, Australia

${ }^{2}$ Kids Neuroscience Centre, The Children's Hospital at Westmead, Faculty of Medicine and Health, University of Sydney, Westmead, NSW, Australia

Full list of author information is available at the end of the article
}

presentation, progression, and outcomes of ASD can be largely heterogeneous, posing difficulties for identification and treatment ${ }^{3-5}$. The causes of ASD are unknown; genetic, epigenetic, and environmental factors have all been implicated ${ }^{6,7}$.

Increasing evidence suggests that immune system dysregulation $^{8-11}$, particularly maternal immune activation $(\mathrm{MIA})^{12-14}$, is one factor that may be involved in the pathophysiology of ASD and other neurodevelopmental disorders. An extensive body of preclinical research has provided substantial evidence for the MIA hypothesis in $\mathrm{ASD}^{15-18}$. Human epidemiological studies also link chronic immune/inflammatory conditions in mothers, such as autoimmune conditions ${ }^{19-26}$, and asthma/allergic 
conditions $^{27-29}$, with increased risk of ASD and other neurodevelopmental disorders in offspring. Cohort studies have shown that autoimmune conditions are more prevalent in mothers of children with $\mathrm{ASD}^{23}$, particularly in cases of ASD with combined developmental delay ${ }^{24}$ or regression ${ }^{25}$. Similarly, maternal asthma has been linked with increased risk of ASD with combined intellectual disability in offspring ${ }^{29}$. Maternal asthma and allergies have also been associated with increased social impairment symptoms in children with $\mathrm{ASD}^{30}$.

Autoimmune conditions, asthma, and allergies involve activation of inflammatory pathways and elevated levels of cytokines and chemokines ${ }^{31-35}$. It has been posited that prenatal exposure to inflammatory conditions interferes with neurodevelopment and foetal programming, leading to adverse outcomes in offspring ${ }^{36,37}$. Many cytokines and chemokines can cross the blood-brain barrier and the placenta, both potential avenues through which foetal development can be disrupted ${ }^{38-40}$. However, cytokines do not necessarily need to cross these barriers to elicit their effects; they can bind receptors at the placental interface leading to downstream effects on the placenta and foetus. The role of cytokines in the pathophysiology of ASD is further supported by elevated levels of proinflammatory cytokines and chemokines found at birth or during development in at least a subset of individuals with ASD that has been correlated with increased severity of ASD symptoms ${ }^{41-47}$.

Many animal studies have found that MIA predominantly affects males, compared to females, in the domains of repetitive behaviour ${ }^{48,49}$, motor development $^{50}$, and learning and memory ${ }^{51,52}$. These findings are reflected in the incidence rate of ASD with four times more human males ${ }^{1}$ being affected and higher rates of repetitive behaviour are found in males, compared to females ${ }^{53,54}$. A recent study in humans also showed that the child's sex, along with gestational timing of maternal inflammation (measured by elevated pro-inflammatory cytokines), can contribute to differential behavioural outcomes in the child ${ }^{55}$. While males appear to be more vulnerable to a maternal inflammation-mediated expression of neurodevelopmental difficulties, the mechanisms underlying this association remain unclear. Moreover, ASD was not specifically investigated in this study. Offspring sex likely interacts with other factors, such as the type of maternal activation (e.g. infection vs. chronic diseases such as autoimmunity, or asthma), the timing (i.e. early vs. late gestation), the duration (short vs. episodic/ continuous exposures), and severity of the MIA, to produce differential behavioural outcomes in males and females $^{55-57}$.

The existing evidence suggests a possible link between maternal immune conditions and the observed ASD phenotype in offspring. Phenotypic outcomes in response to the presence of maternal immune conditions may vary by offspring sex. In this study, we explored whether maternal immune conditions (autoimmune, asthma, and other chronic immune/inflammatory conditions) were associated with increased severity of adverse outcomes in a large, well-characterized cohort of preschool-aged children with ASD. We compared the severity of ASD, behavioural and emotional well-being, and cognitive functioning between male and female children to explore the role of offspring sex in following exposures to maternal immune conditions. We hypothesized that children whose mothers had a history of immune/ inflammatory conditions would have increased severity of ASD and behavioural and emotional problems, along with decreased cognitive function. Notably, this sample contains a relatively large proportion of females $(n=111)$, allowing for examination of sex differences.

\section{Methods \\ Participants}

Participants included mothers and children who were enrolled through the Autism Phenome Project (APP) or Girls with Autism Imaging of Neurodevelopment (GAIN) study, conducted at the University of California Davis MIND Institute. The GAIN study has an identical study design to the APP and enriches the predominantly male APP cohort with additional female participants. The study protocols, including recruitment and behavioural assessments for the APP and GAIN studies, have been previously described in detail ${ }^{58-60}$. All assessment measures described below were conducted as part of the APP and GAIN study protocols during enrolment in these studies. Children with a community diagnosis of ASD were included in this study $(n=363$; 252 males, 111 females; median age 3.07 [interquartile range 2.64-3.36 years]). This ASD diagnosis was confirmed upon enrolment using the Autism Diagnostic Interview-Revised ${ }^{61}$ and the Autism Diagnostic Observation Schedule $(\mathrm{ADOS})^{62}$. The presence of Fragile X syndrome or other neurological (e.g. seizures), psychiatric, or medical conditions were considered exclusion criteria for all children. The administration of all diagnostic instruments was carried out by experienced clinicians at the MIND Institute. All participants were English speakers, ambulatory, and had no suspected vision or hearing problems. All participants were screened via a parental interview for current and past physical illness. This study was approved by the institutional review board at the University of California, Davis. Informed consent was obtained before participation.

\section{ASD phenotype and offspring outcomes}

ASD severity was measured using ADOS calibrated severity scores (CSSs). CSSs were calculated for the 
subdomains of social affect (CSS-SA) and restricted and repetitive behaviour, as well as an overall CSS. The ADOS CSSs are based on a scale on 1-10, where 1 represents minimal evidence of ASD-related symptoms and 10 denotes a high severity of symptoms ${ }^{63,64}$.

Developmental performance and cognitive functioning were assessed using the Mullen Scales of Early Learning (MSEL). The MSEL has components for visual reception, fine motor, receptive language, and expressive language. A developmental quotient (DQ) was calculated as the average of the age equivalent subscale scores divided by the chronological age at the time of testing and multiplied by 100 . DQs provide a consistent metric for cognitive measures and accommodate floor effects. The nonverbal DQ (NVDQ) includes the visual reception and fine motor subscales, while the verbal DQ (VDQ) includes the receptive and expressive language subscales.

The Child Behaviour Checklist $(\mathrm{CBCL})^{65}$, a widely used and well-accepted measure of child psychopathology ${ }^{66,67}$, was used to assess behavioural and emotional problems in children. The CBCL is a parent-rated questionnaire which contains a list of 100 behavioural/emotional problem items that parents rate as: not true, somewhat or sometimes true, or very or often true of their children. The CBCL produces raw scores that were transformed into three summary $\mathrm{T}$ scores (standardized by age and sex): (a) Total behaviour; (b) Externalizing (delinquency, aggression) behaviour; and (c) Internalizing (withdrawal, somatic complaints, anxious/depressed) behaviour; these were analysed as continuous variables referred to as 'scores'. In addition, we analysed children who had CBCL T scores $>60$, which is an established threshold of clinically significant level of concern ${ }^{65}$, referred to as 'morbidity'.

\section{Maternal immune history}

Family medical history data, including the presence of maternal autoimmune conditions, chronic allergic/atopic conditions such as asthma, allergies, and eczema, and other inflammatory diseases was evaluated via a physician interview with the participant's parent. Physicians were provided with a list of immune/autoimmune/inflammatory conditions to make this determination. Based on this information, mothers (and their children) were classified into two groups: maternal immune (one or more of the above immune-related conditions) and maternal non-immune.

\section{Statistical analysis}

Demographic characteristics of mothers and children in the maternal immune and maternal non-immune groups were compared using independent-samples $t$ tests, Mann-Whitney $U$ tests, and Chi-square tests, as appropriate. Generalized linear models (normal distribution) were used to investigate the effect of maternal immune status on the continuous CBCL, MSEL, and ADOS scores, generating $\beta$ coefficients and $95 \%$ confidence intervals (CIs). Generalized linear models (binomial distribution with logit link) were used to investigate CBCL morbidity ( $\mathrm{T}$ score $>60$ ) on the Total, Internalizing, and Externalizing scales, generating odds ratios (ORs) and 95\% CIs. All models were adjusted for maternal age at childbirth and offspring sex. In addition, generalized linear models (adjusted only for maternal age at childbirth) were used separately in males and females to evaluate whether sex played a role in outcome differences between children in the maternal immune and maternal non-immune groups. Participants with missing data for an outcome measure were excluded from the analysis for that measure. There was a variable percentage of missing data in each measure for the cohort: CBCL Internalizing 14\%, CBCL Externalizing 12\%, CBCL Total 23\%, MSEL VDQ 3\%, MSEL NVDQ 3\%, MSEL DQ 2\%, and ADOS all scales 3\%. Statistical analyses were performed using IBM SPSS Statistics 26 and graphs were created using GraphPad Prism 8.

\section{Results}

\section{Characteristics of the maternal immune and maternal non- immune groups}

Of the total 363 mothers included in the study, immune conditions of any type were found in $99(27.27 \%)$ mothers (maternal immune group). The remaining 246 (72.73\%) mothers who did not report any immune conditions served as a comparison group (maternal non-immune group). There were no significant differences between the maternal immune and maternal non-immune groups in the level of maternal education, annual household income, maternal age, and offspring race (Table 1). The median age of children in the maternal immune group was slightly lower than those in the maternal nonimmune group (2.98 vs. 3.15 years, $p=0.0133$; Table 1 ).

Within the maternal immune group, asthma was the most common condition, found in 63 mothers $(23.95 \%$ of total). Other frequent conditions included Hashimoto's thyroiditis $(n=17)$, Raynaud's disease $(n=10)$, alopecia $(n=5)$, psoriasis $(n=5)$, and rheumatoid arthritis $(n=4$; Supplementary Table 1). Other immune conditions, each reported by 1-2 mothers in the immune group, are shown in Supplementary Table 1. A history of maternal immune conditions was more common in male children with ASD (31.3\% of total) compared to female children (18.0\%; $\chi^{2}(1)=6.904, p=0.009$; Fig. 1a). Specifically, a history of maternal asthma was twice as common in male children (20.2\%) compared to female children (10.8\%) with ASD $\left(\chi^{2}(1)=4.775, p=0.029\right.$; Fig. 1b).

\section{Offspring outcomes}

Maternal immune history was examined as a predictor of symptom outcomes in offspring by comparing the maternal immune and maternal non-immune groups. 
Table 1 Demographic characteristics of the mothers and children in the maternal immune and maternal non-immune groups $(n=363)$.

\begin{tabular}{|c|c|c|c|c|}
\hline & Full cohort & Maternal immune & Maternal non-immune & $p$ value $^{\mathrm{a}}$ \\
\hline Number of participants, $n$ (\%) & 363 & 99 & 264 & \\
\hline Mother's education, $n(\%)^{\mathrm{b}}$ & & & & 0.644 \\
\hline High school graduate or less & $26(7.16)$ & $4(4.04)$ & $22(8.3)$ & \\
\hline Technical/vocational & $37(10.19)$ & $12(12.12)$ & $25(9.47)$ & \\
\hline Some college credit & $59(16.25)$ & $19(19.19)$ & $40(15.15)$ & \\
\hline Associate degree & $47(12.95)$ & $12(12.12)$ & $35(13.26)$ & \\
\hline Bachelor's degree & $96(26.45)$ & $29(29.29)$ & $67(25.38)$ & \\
\hline Professional, Master's, or Doctorate degree & $47(12.95)$ & $15(15.15)$ & $32(12.12)$ & \\
\hline Annual family income, $n(\%)^{b}$ & & & & 0.730 \\
\hline$\leq \$ 29000$ & $38(10.4)$ & $8(8.08)$ & $30(11.36)$ & \\
\hline$\$ 30,000-\$ 49,000$ & $44(12.12)$ & $15(15.15)$ & $29(10.98)$ & \\
\hline$\$ 50,000-\$ 74,999$ & $54(14.88)$ & $17(17.17)$ & $37(14.02)$ & \\
\hline$\$ 75,000-\$ 99,999$ & $59(15.25)$ & $18(18.18)$ & $41(15.53)$ & \\
\hline$\$ 100,000-\$ 149,999$ & $48(13.22)$ & $11(11.11)$ & $37(14.02)$ & \\
\hline$\geq \$ 150,000$ & $48(13.22)$ & $14(14.14)$ & $34(12.88)$ & \\
\hline Mother's age at childbirth, mean (SD) & $31.57(5.35)$ & $31.87(4.87)$ & $31.46(5.53)$ & 0.528 \\
\hline Offspring race, $n(\%)^{\mathrm{b}}$ & & & & 0.517 \\
\hline Asian & 29 (7.99) & $7(7.07)$ & $22(8.33)$ & \\
\hline Mixed & $41(11.29)$ & $11(11.11)$ & $30(11.36)$ & \\
\hline Other & $31(8.54)$ & $6(6.06)$ & $25(6.87)$ & \\
\hline White/Caucasian & 229 (63.09) & $71(71.72)$ & $158(59.85)$ & \\
\hline Offspring age, median (IQR) & $3.07(2.64-3.36)$ & $2.98(2.53-3.23)$ & $3.15(2.70-3.38)$ & $0.013^{c}$ \\
\hline Offspring sex, $n(\%)$ & & & & 0.009 \\
\hline Male & $252(69.42)$ & $79(79.80)$ & $173(65.53)$ & \\
\hline Female & $111(30.58)$ & $20(20.20)$ & $91(34.47)$ & \\
\hline
\end{tabular}

$S D$ standard deviation, IQR interquartile range.

${ }^{a} p$ Values are reported for comparisons between the maternal immune and maternal non-immune groups using independent-samples $t$ tests, Mann-Whitney $U$ test, or Chi-square tests as appropriate.

${ }^{b}$ Percentages expressed as a fraction of the number of participants in each group. Percentages may not total 100 due to missing data for some variables. ${ }^{c}$ Mann-Whitney $U$ test used for offspring age as data were not normally distributed.
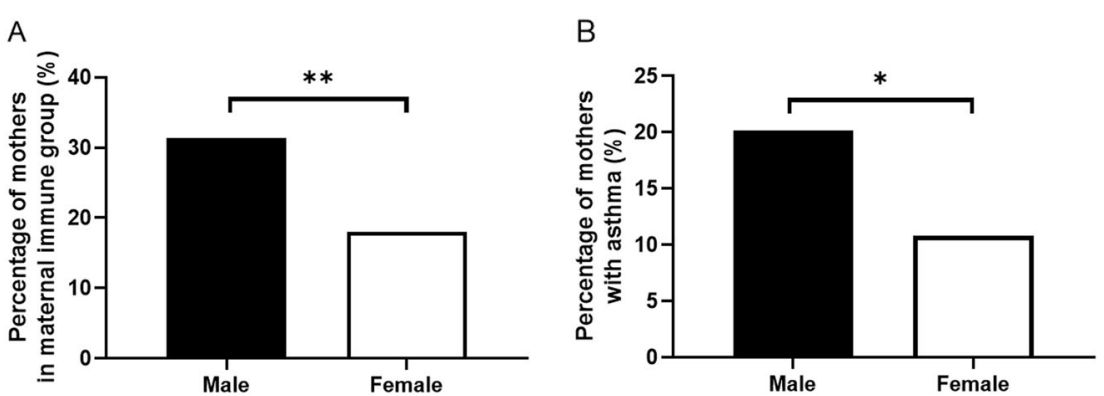

Fig. 1 History of maternal immune conditions and asthma in male and female children with ASD. Percentage of mothers with any immune conditions (a) and asthma (b) were significantly higher in male children than female children. 
Table 2 Descriptive statistics for offspring CBCL, MSEL, and ADOS scores in the maternal immune and maternal nonimmune groups.

\begin{tabular}{lccc}
\hline & Full cohort $^{\text {a }}$ & Maternal immune group & Maternal non-immune group \\
\hline CBCL Internalizing, mean (SD) & $62.05(9.33)$ & $62.95(8.50)$ & $61.72(9.62)$ \\
CBCL Externalizing, mean (SD) & $59.01(10.61)$ & $60.36(10.47)$ & $58.49(10.65)$ \\
CBCL Total, mean (SD) & $62.23(10.54)$ & $64.01(9.81)$ & $61.89(10.76)$ \\
CBCL Internalizing >60, n (\%) & $184(50.69)$ & $56(65.88)$ & $128(56.39)$ \\
CBCL Externalizing >60, $n$ (\%) & $139(38.29)$ & $47(53.41)$ & $92(40.00)$ \\
CBCL Total >60, n (\%) & $164(45.18)$ & $53(69.74)$ & $111(54.41)$ \\
MSEL VDQ, mean (SD) & $56.56(25.25)$ & $61.13(24.81)$ & $54.80(25.25)$ \\
MSEL NVDQ, mean (SD) & $69.74(19.00)$ & $75.11(18.62)$ & $67.68(18.78)$ \\
MSEL DQ, mean (SD) & $63.17(20.64)$ & $68.19(19.61)$ & $61.24(20.74)$ \\
ADOS CSS-SA, mean (SD) & $6.99(1.67)$ & $6.91(1.77)$ & $7.02(1.64)$ \\
ADOS CSS-RRB, mean (SD) & $8.42(1.53)$ & $8.30(1.46)$ & $8.46(1.56)$ \\
ADOS CSS, mean (SD) & $7.56(1.72)$ & $7.47(1.77)$ & $7.60(1.70)$
\end{tabular}

SD standard deviation, CBCL Child Behaviour Checklist, MSEL Mullens Scales of Early Learning, VDQ verbal developmental quotient, NVDQ nonverbal developmental quotient, $D Q$ developmental quotient, $A D O S$ Autism Diagnostic Observation Schedule, CSS calibrated severity score, SA social affect, RRB restricted repetitive behaviour.

a Percentage of missing data in each measure for the full cohort: CBCL Internalizing 14\%, CBCL Externalizing 12\%, CBCL Total 23\%, MSEL VDQ 3\%, MSEL NVDQ 3\%, MSEL DQ $2 \%$, ADOS all scales $3 \%$.

Owing to the high frequency of maternal asthma in the immune group, separate analyses were also conducted to examine maternal asthma alone as a predictor of offspring outcomes. In these analyses, mothers with asthma were compared with all mothers without asthma from both the maternal immune and maternal non-immune groups. The means and standard deviations of the CBCL, MSEL, and ADOS scores in the maternal immune and maternal nonimmune groups are presented in Table 2.

Regarding behaviour, children in the maternal immune group had significantly higher CBCL scores on the Externalizing $(p=0.018)$ and Total $(p=0.021)$ scales (Table 3$)$. When examining separately maternal asthma, there was also a significant association with increased Externalizing scores $(p=0.045)$ and risk of more impaired behaviour on the Externalizing scale ( $p=0.007$; Table 3$)$. Maternal immune conditions were not associated with decreased cognition; significantly higher NVDQ $(p=0.029)$ and DQ $(p=0.001)$ scores were found on the MSEL assessment (Table 3). By contrast, there was no significant effect of maternal asthma alone and cognition on MSEL scores (Table 3). Regarding observational ADOS autism assessment, there were no significant effects of maternal immune conditions or maternal asthma on ADOS CSS scores (Table 3).

The effects of maternal immune conditions on offspring outcomes, stratified by offspring sex, were next assessed (Table 4). Specifically, maternal immune conditions were associated with significantly increased Total
CBCL scores $(p=0.026)$ and increased risk of impairment on the Externalizing scale $(p=0.045)$ in females (Table 4). Maternal asthma alone was associated with significantly increased risk of behavioural morbidity on the Externalizing scale in both males $(p=0.035)$ and females $(p=0.035$; Table 4$)$.

Females in the maternal immune group had significantly increased scores on all scales of the MSEL (VDQ $p=0.006$, NVDQ $p=0.024$, DQ $p=0.006$; Table 4 ); however, no significant differences were found in males, suggesting that improvement was driven by females in this study. Maternal asthma alone did not have any significant effects on male or female MSEL scores (Table 4). In the maternal immune group, females showed a small, significant decrease in ADOS CSS-SA scores $(p=$ 0.015; Table 4) suggesting better sociability, but male ADOS scores were not significantly affected. Maternal asthma alone did not significantly affect the ADOS scores in males or females (Table 4).

\section{Discussion}

This study investigated whether maternal immune conditions (autoimmune, asthma, and other chronic immune/inflammatory conditions) influenced phenotypic outcomes in children with ASD. Further, we explored whether offspring sex interacts with the presence of maternal immune conditions to influence behavioural outcomes in children. In this cohort, a history of maternal immune conditions was more common in male children 
Table 3 General linear models showing maternal immune conditions and maternal asthma as predictors of offspring outcomes.

\begin{tabular}{|c|c|c|}
\hline & Maternal immune group & Maternal asthma only \\
\hline CBCL Internalizing, $\beta[95 \% \mathrm{Cl}]$ & $1.66[-0.69,4.01] ; p=0.166$ & $2.28[-0.48,2.62] ; p=0.106$ \\
\hline CBCL Externalizing, $\beta$ [95\% Cl] & $2.10[-0.50,4.71] ; p=0.113$ & $3.09[0.07,6.12] ; p=0.045$ \\
\hline CBCL Total, $\beta[95 \% \mathrm{Cl}]$ & $2.28[-0.51,5.07] ; p=0.109$ & $2.45[-0.84,5.74] ; p=0.114$ \\
\hline CBCL Internalizing >60, OR $[95 \% \mathrm{Cl}]$ & $1.70[0.99,2.92] ; p=0.54$ & $1.71[0.90,3.24] ; p=0.104$ \\
\hline CBCL Externalizing >60, OR $[95 \% \mathrm{Cl}]$ & $1.87[1.11,3.15] ; p=0.018$ & $2.33[1.26,4.28] ; p=0.007$ \\
\hline CBCL Total >60, OR $[95 \% \mathrm{Cl}]$ & $1.98[1.11,3.53] ; p=0.021$ & $1.88[0.94,3.74] ; p=0.074$ \\
\hline MSEL VDQ, $\beta[95 \% \mathrm{Cl}]$ & $2.03[-0.45,4.50] ; p=0.108$ & $0.57[-2.33,3.48] ; p=0.699$ \\
\hline MSEL NVDQ, $\beta[95 \% \mathrm{CI}]$ & $6.75[0.68,12.82] ; p=0.029$ & $1.48[-5.67,8.64] ; p=0.685$ \\
\hline MSEL DQ, $\beta[95 \% \mathrm{Cl}]$ & $7.69[3.18,12.20] ; p=0.001$ & $4.04[-1.31,9.39] ; p=0.139$ \\
\hline ADOS CSS-SA, $\beta[95 \% \mathrm{Cl}]$ & $-0.13[-0.53,0.27] ; p=0.526$ & $0.10[-0.37,0.57] ; p=0.668$ \\
\hline ADOS CSS-RRB, $\beta[95 \% \mathrm{Cl}]$ & $-0.11[-0.48,0.27] ; p=0.579$ & $-0.08[-0.52,0.36] ; p=0.721$ \\
\hline $\operatorname{ADOS} \mathrm{CSS}, \beta[95 \% \mathrm{Cl}]$ & $-0.11[-0.52,0.32] ; p=0.625$ & $0.11[-0.38,0.60] ; p=0.657$ \\
\hline
\end{tabular}

OR odds ratio, Cl confidence interval, CBCL Child Behaviour Checklist, MSEL Mullens Scales of Early Learning, VDQ verbal developmental quotient, NVDQ nonverbal developmental quotient, $D Q$ developmental quotient, $A D O S$ Autism Diagnostic Observation Schedule, CSS calibrated severity score, SA social affect, RRB restricted repetitive behaviour.

with ASD compared to female children. Maternal asthma, the most frequently reported immune condition, was also more common in male compared to female children. While previous studies have shown that maternal immune conditions are more prevalent in mothers of children with $\mathrm{ASD}^{23-25}$, our results suggest that this may be influenced by offspring sex, a finding not described previously. These results are consistent with animal research suggesting that males are more vulnerable to more neurodevelopmental abnormalities after MIA $^{48-52}$.

Maternal immune conditions and maternal asthma were associated with increased CBCL scores and more impairment in children, particularly on the Externalizing and Total scales. The effect of maternal immune conditions was seen in female children only; however, the effect of maternal asthma was present in both male and female children. Overall, the results suggest that the presence of maternal immune conditions and maternal asthma may be linked to increased severity of behavioural and emotional problems in both male and female children with ASD.

These findings align with a recent study in humans which showed that elevated maternal cytokine levels in the first and second trimesters of pregnancy were associated with increased internalizing and externalizing symptoms in children. This study also found that gestational timing of inflammation and the child's sex can influence behavioural outcomes ${ }^{55}$. The current study did not consider gestational timing; however, the immune conditions included here are chronic, rather than acute, in nature. Furthermore, the current study focussed specifically on behavioural outcomes in children with ASD, while the previous study explored outcomes in a general cohort. Maternal asthma and allergies have also been linked to increased social impairments in an Australian cohort studying children with $\mathrm{ASD}^{30}$, providing further support for the notion that maternal immune conditions, or in particular atopic/asthma immune activation, may influence behavioural and emotional outcomes in children in ASD. In a second large population-based cohort in the US, maternal asthma was associated with risk of ASD, with the children with ASD exhibiting more severe impairments ${ }^{29}$.

By contrast, maternal immune conditions were not associated with decreased cognitive functioning. Rather, maternal immune conditions were linked to higher NVDQ and DQ scores in the full cohort. However, this effect was not seen when examining the maternal asthma group separately. Furthermore, when analysed by sex, this improved cognition was only seen in females, who had higher scores on all three MSEL scales. These findings contrast with previous studies which showed that maternal autoimmune conditions were linked to ASD with combined intellectual disability and developmental delay $^{24,29}$. However, these studies did not investigate male and female differences. The female specificity of high MSEL scores may demonstrate that, in cases of ASD where maternal immune conditions are present, female offspring are less likely to be susceptible to adverse cognitive outcomes in response to maternal inflammation than male offspring. Of note, animal studies support this hypothesis, showing that MIA detrimentally affects males, compared to females, in the domains of learning and memory $^{51,52}$. 


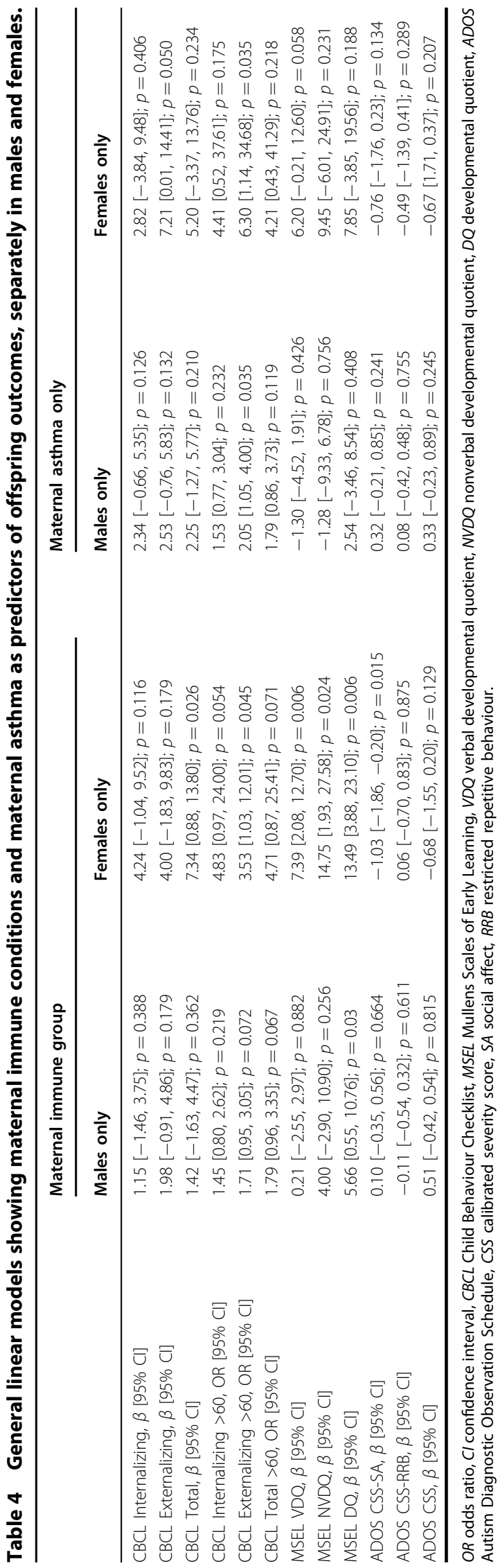

Of interest, the ADOS scores remained largely unaffected by maternal immune conditions, with only a small decrease found in females on the social affect domain. This finding is consistent with the notion that, in cases of ASD where maternal immune conditions are present, female children are less vulnerable to the detrimental effects of maternal inflammation. It is important to note that this cohort includes preschool-age children with an existing community diagnosis of ASD. It is possible that this sample is biased by children with more severe ASD as children with milder symptoms may not be diagnosed until a later age. Further studies, representing a broader spectrum of ASD, are required to determine whether maternal immune conditions influence the severity of ASD symptoms.

Overall, the results indicate that the presence of maternal immune conditions, particularly maternal asthma, may influence the phenotypic expression of ASD. In addition, offspring sex may contribute to the differential outcomes observed between male and female children in the maternal immune and maternal non-immune groups. Increasing evidence from preclinical models suggests that male offspring are more susceptible to adverse outcomes as a result of MIA compared to female offspring $^{48-52}$. Although these models typically rely on short-term exposures to infections rather than potentially ongoing or episodic activation from chronic maternal immune conditions, the preclinical observations support findings seen in this study. While the findings of this study do not directly show greater impairments in male children, they do suggest that sex differences are an important factor in exposures from maternal immune conditions. Thus maternal immune conditions may be one environmental factor that contributes to the higher male prevalence seen in ASD.

The mechanisms underlying MIA and its conferred risk to offspring neurodevelopment are likely to be complex and multi-faceted. Epigenetic regulation of existing genetic vulnerabilities by MIA and other environmental factors likely places the foetus at greater risk for adverse outcomes ${ }^{68-70}$. Cytokines, chemokines, and antibodies, which are activated as a result of MIA, may interfere with foetal programming and development ${ }^{36}$. Microglia, the resident immune cells of the brain, have also emerged as key mediators of MIA ${ }^{71-74}$. Abnormalities in microglia phenotype, which can lead to disrupted synaptic pruning, have been associated with ASD and MIA ${ }^{73,75,76}$.

There are a number of potential limitations to this study. The data regarding maternal immune conditions was collected when children were enrolled in the study, not during pregnancy. It is possible that some mothers in the maternal immune group may not have had active immune conditions during gestation. The potential for recall bias in reporting notwithstanding, the presence of 
these conditions infers an immune dysregulation predisposition. Moreover, since the children were young at enrolment and given the chronic nature of the reported conditions, we suspect that underlying immune aberrations are likely to have been present in the mother during pregnancy ${ }^{77-79}$. Although there was a range of autoimmune conditions and other syndromes of inflammation reported in the maternal immune group, the majority of the mothers reported asthma. A further limitation of the study is that it is difficult to make inferences about many of the specific immune conditions other than asthma, especially those reported by only a few mothers. This study did not have data to directly investigate the influence of maternal infections during pregnancy, another important type of MIA, on child outcomes. Other variables that may contribute to inflammation or interact with existing conditions, such as obesity, diet, and stress, were also not collected ${ }^{80,81}$. There are other variables that could influence offspring outcomes which were not considered here, such as psychiatric conditions in mothers, recall bias in self-report of immune conditions, and use of medications during pregnancy.

The type and timing of MIA during pregnancy has been noted as an important factor which might exert differential effects on neurodevelopment ${ }^{55,82,83}$. Different types of MIA may utilize different immune molecules and pathways. For example, maternal asthma is characterized by $\mathrm{T}$ helper $2\left(\mathrm{~T}_{\mathrm{H}} 2\right)$ cell-mediated humoral responses ${ }^{77,84}$, whereas viral infections/bacterial infections are typically cell-mediated or $\mathrm{T}_{\mathrm{H}} 1$ responses ${ }^{85}$. Autoimmunity could be both $\mathrm{T}_{\mathrm{H}} 1$ and $\mathrm{T}_{\mathrm{H}} 2$, depending on the disease ${ }^{34}$. Innate immune responses, such as cytokine production, are also shared between asthma, autoimmunity, and infections $^{32,35}$. In terms of the MIA animal models, different immune activators have been used to investigate different pathways, including the traditional infection-based model, as well as other pro-inflammatory states, such as asthma, obesity, and stress. However, based on this data, a clear finding was that immune activation caused by maternal asthma increases the risk for adverse offspring outcomes, providing clues to potential immune pathways that may be involved, such as the $\mathrm{T}_{\mathrm{H}} 2$ pathways.

Further research is warranted in humans to better understand the influence of maternal immune conditions on outcomes in children with ASD. Future studies should aim to comprehensively characterize immune activation in mothers during pregnancy, including the type, severity, and gestational timing of immune conditions, and then longitudinally examine offspring outcomes. Studies involving biomarkers and immunological profiling of both mothers and offspring are required to unravel the molecular mechanisms underpinning MIA. Such studies would enable clinical and biological characterization of immune- mediated subtypes in ASD. This may allow for the identification of therapeutic targets and exploration of immune-focussed medical treatments for subgroups of children with ASD. Furthermore, addressing mechanisms of maternal inflammation at the level of prenatal programming may lead to the development of prevention strategies for ASD.

This study shows that maternal immune conditions, particularly maternal asthma, are associated with increased behavioural and emotional problems in children with ASD. Furthermore, offspring sex may interact with maternal immune conditions to influence offspring outcomes in ASD, particularly in terms of cognition. This study adds to a growing body of literature which highlights that maternal immune conditions are an important factor in the phenotypic expression of ASD. Characterizing clinical associations between maternal immune conditions and symptom outcomes in children is an essential step in understanding the pathophysiology of ASD and has important implications for diagnosis and treatment.

\section{Acknowledgements}

We would like to acknowledge and thank the families and children who participated in the GAIN and APP studies. Funding for this study was provided by the National Institute of Health (RO1MH118209, RO1HD090214,

R21MH116383, R21ES025560 [to P.A.]; RO1MH104438 [to C.W.N.]), Autism Speaks Foundation, Autism Research Institute, Grace Gardner Johnson and Jane B. Johnson, CTSC Pilot Translational and Clinical Studies Program, A Child/ Lifespan Health Award [to P.A.], and the UC Davis MIND Institute.

\section{Author details \\ ${ }^{1}$ Autism Clinic for Translational Research, Brain and Mind Centre, Children's Hospital Westmead Clinical School, Faculty of Medicine and Health, University of Sydney, Camperdown, NSW, Australia. ${ }^{2}$ Kids Neuroscience Centre, The Children's Hospital at Westmead, Faculty of Medicine and Health, University of Sydney, Westmead, NSW, Australia. ${ }^{3}$ Department of Medical Microbiology and Immunology and MIND Institute, UC Davis, Davis, CA, USA. ${ }^{4}$ Department of Psychiatry and MIND Institute, UC Davis, Davis, CA, USA}

Conflict of interest

The authors declare that they have no conflict of interest.

\section{Publisher's note}

Springer Nature remains neutral with regard to jurisdictional claims in published maps and institutional affiliations.

Supplementary Information accompanies this paper at (https://doi.org/ 10.1038/s41398-020-00976-2).

Received: 5 May 2020 Revised: 21 July 2020 Accepted: 24 July 2020 Published online: 14 August 2020

\footnotetext{
References

1. Baio, J. et al. Prevalence of autism spectrum disorder among children aged 8 years - Autism and Developmental Disabilities Monitoring Network, 11 Sites, United States, 2014. MMWR Surveill. Summ. 67, 1-23 (2018).

2. American Psychiatric Association. Diagnostic and Statistical Manual of Mental Disorders 5 edn (American Psychiatric Association, Arlington, VA, 2013).

3. Howlin, P., Goode, S., Hutton, J. \& Rutter, M. Adult outcome for children with autism. J. Child Psychol. Psychiatry 45, 212-229 (2004).
} 
4. Szatmari, P. et al. Developmental trajectories of symptom severity and adaptive functioning in an inception cohort of preschool children with autism spectrum disorder. JAMA Psychiatry 72, 276-283 (2015).

5. Bryson, S. E., Rogers, S. J. \& Fombonne, E. Autism spectrum disorders: early detection, intervention, education, and psychopharmacological management. Can. J. Psychiatry 48, 506-516 (2003).

6. Persico, A. M. \& Bourgeron, T. Searching for ways out of the autism maze: genetic, epigenetic and environmental clues. Trends Neurosci. 29, 349-358 (2006).

7. Gentile, I. et al. Etiopathogenesis of autism spectrum disorders: fitting the pieces of the puzzle together. Med. Hypotheses 81, 26-35 (2013).

8. Ashwood, P., Wills, S., Van \& de Water, J. The immune response in autism: a new frontier for autism research. J. Leukoc. Biol. 80, 1-15 (2006).

9. McDougle, C. J. et al. Toward an immune-mediated subtype of autism spectrum disorder. Brain Res. 1617, 72-92 (2015).

10. Noriega, D. B. \& Savelkoul, H. F. Immune dysregulation in autism spectrum disorder. Eur. J. Pediatr. 173, 33-43 (2014).

11. Hughes, H. K., Mills, Ko. E., Rose, D. \& Ashwood, P. Immune dysfunction and autoimmunity as pathological mechanisms in autism spectrum disorders. Front. Cell Neurosci. 12, 405 (2018).

12. Estes, M. L. \& McAllister, A. K. Maternal immune activation: Implications for neuropsychiatric disorders. Science 353, 772-777 (2016).

13. Knuesel, I. et al. Maternal immune activation and abnormal brain development across CNS disorders. Nat. Rev. Neurol. 10, 643-660 (2014).

14. Boulanger-Bertolus, J., Pancaro, C. \& Mashour, G. A. Increasing role of maternal immune activation in neurodevelopmental disorders. Front. Behav. Neurosci. 12, 230 (2018).

15. Careaga, M., Murai, T. \& Bauman, M. D. Maternal immune activation and autism spectrum disorder: from rodents to nonhuman and human primates. Biol. Psychiatry 81, 391-401 (2017).

16. Malkova, N. V., Yu, C. Z., Hsiao, E. Y., Moore, M. J. \& Patterson, P. H. Maternal immune activation yields offspring displaying mouse versions of the three core symptoms of autism. Brain Behav. Immun. 26, 607-616 (2012).

17. Schwartzer, J. J., Careaga, M., Chang, C., Onore, C. E. \& Ashwood, P. Allergic fetal priming leads to developmental, behavioral and neurobiological changes in mice. Transl. Psychiatry 5, e543 (2015).

18. Schwartzer, J. J., Careaga, M., Coburn, M. A., Rose, D. R. \& Ashwood, P. Behavioral impact of maternal allergic-asthma in two genetically distinct mouse strains. Brain Behav. Immun. 63, 99-107 (2017).

19. Atladottir, H. O. et al. Association of family history of autoimmune diseases and autism spectrum disorders. Pediatrics 124, 687-694 (2009).

20. Chen, S. W. et al. Maternal autoimmune diseases and the risk of autism spectrum disorders in offspring: A systematic review and meta-analysis. Behav. Brain Res. 296, 61-69 (2016).

21. Keil, A. et al. Parental autoimmune diseases associated with autism spectrum disorders in offspring. Epidemiology 21, 805-808 (2010).

22. $\mathrm{Wu}, \mathrm{S}$. et al. Family history of autoimmune diseases is associated with an increased risk of autism in children: a systematic review and meta-analysis. Neurosci. Biobehav Rev. 55, 322-332 (2015).

23. Comi, A. M., Zimmerman, A. W., Frye, V. H., Law, P. A. \& Peeden, J. N. Familial clustering of autoimmune disorders and evaluation of medical risk factors in autism. J. Child Neurol. 14, 388-394 (1999).

24. Lyall, K., Ashwood, P., Van de Water, J. \& Hertz-Picciotto, I. Maternal immunemediated conditions, autism spectrum disorders, and developmental delay. J. Autism Dev. Disord. 44, 1546-1555 (2014).

25. Scott, O., Shi, D., Andriashek, D., Clark, B. \& Goez, H. R. Clinical clues for autoimmunity and neuroinflammation in patients with autistic regression. Dev. Med. Child Neurol. 59, 947-951 (2017).

26. Jones, H. F. et al. Maternal thyroid autoimmunity associated with acute-onset neuropsychiatric disorders and global regression in offspring. Dev. Med. Child Neurol. 61, 984-988 (2019).

27. Croen, L. A. et al. Maternal autoimmune diseases, asthma and allergies, and childhood autism spectrum disorders: a case-control study. Arch. Pediatr. Adolesc. Med. 159, 151-157 (2005).

28. Gong, T. et al. Parental asthma and risk of autism spectrum disorder in offspring: a population and family-based case-control study. Clin. Exp. Allergy 49, 883-891 (2019).

29. Croen, L. A. et al. Family history of immune conditions and autism spectrum and developmental disorders: findings from the study to explore early development. Autism Res. 12, 123-135 (2019).
30. Patel, S. et al. Social impairments in autism spectrum disorder are related to maternal immune history profile. Mol. Psychiatry 23, 1794-1797 (2018).

31. Kuo, I. H., Yoshida, T., De Benedetto, A. \& Beck, L. A. The cutaneous innate immune response in patients with atopic dermatitis. J. Allergy Clin. Immunol. 131, 266-278 (2013).

32. Ngoc, P. L., Gold, D. R., Tzianabos, A. O., Weiss, S. T. \& Celedon, J. C. Cytokines, allergy, and asthma. Curr. Opin. Allergy Clin. Immunol. 5, 161-166 (2005).

33. Newcomb, D. C. \& Peebles, R. S. Jr Th17-mediated inflammation in asthma. Curr. Opin. Immunol. 25, 755-760 (2013).

34. Raphael, I., Nalawade, S., Eagar, T. N. \& Forsthuber, T. G. T cell subsets and their signature cytokines in autoimmune and inflammatory diseases. Cytokine 74, 5-17 (2015).

35. Moudgil, K. D. \& Choubey, D. Cytokines in autoimmunity: role in induction regulation, and treatment. J. Interferon Cytokine Res. 31, 695-703 (2011).

36. Parker-Athill, E. C. \& Tan, J. Maternal immune activation and autism spectrum disorder: interleukin-6 signaling as a key mechanistic pathway. Neurosignals 18, 113-128 (2010).

37. Deverman, B. E. \& Patterson, P. H. Cytokines and CNS development. Neuron 64, 61-78 (2009).

38. Zaretsky, M. V., Alexander, J. M., Byrd, W. \& Bawdon, R. E. Transfer of inflammatory cytokines across the placenta. Obstet. Gynecol. 103, 546-550 (2004).

39. Hsiao, E. Y. \& Patterson, P. H. Activation of the maternal immune system induces endocrine changes in the placenta via IL-6. Brain Behav. Immun. 25 604-615 (2011).

40. Hsiao, E. Y. \& Patterson, P. H. Placental regulation of maternal-fetal interactions and brain development. Dev. Neurobiol. 72, 1317-1326 (2012).

41. Ashwood, P. et al. Elevated plasma cytokines in autism spectrum disorders provide evidence of immune dysfunction and are associated with impaired behavioral outcome. Brain Behav. Immun. 25, 40-45 (2011).

42. Ashwood, P. et al. Associations of impaired behaviors with elevated plasma chemokines in autism spectrum disorders. J. Neuroimmunol. 232, 196-199 (2011).

43. Careaga, M., Rogers, S., Hansen, R. L., Amaral, D. G. \& Ashwood, P. Immune endophenotypes in children with autism spectrum disorder. Biol. Psychiatry 81, 434-441 (2017).

44. Masi, A. et al. Cytokine levels and associations with symptom severity in male and female children with autism spectrum disorder. Mol. Autism 8, 63 (2017).

45. Masi, A. et al. Cytokine aberrations in autism spectrum disorder: a systematic review and meta-analysis. Mol. Psychiatry 20, 440-446 (2015).

46. Molloy, C. A. et al. Elevated cytokine levels in children with autism spectrum disorder. J. Neuroimmunol. 172, 198-205 (2006).

47. Krakowiak, P. et al. Neonatal cytokine profiles associated with autism spectrum disorder. Biol. Psychiatry 81, 442-451 (2017).

48. Xuan, I. C. \& Hampson, D. R. Gender-dependent effects of maternal immune activation on the behavior of mouse offspring. PLOS ONE 9, e104433 (2014).

49. Schwartzer, J. J., Careaga, M., Onore, C. E., Berman, R. F. \& Ashwood, P. Maternal immune activation and strain specific interactions in the development of autism-like behaviors in mice. Transl. Psychiatry 3, e240 (2013).

50. Haida, O. et al. Sex-dependent behavioral deficits and neuropathology in a maternal immune activation model of autism. Transl. Psychiatry 9, 124 (2019).

51. Lante, F. et al. Neurodevelopmental damage after prenatal infection: role of oxidative stress in the fetal brain. Free Radic. Biol. Med. 42, 1231-1245 (2007).

52. Howland, J. G., Cazakoff, B. N. \& Zhang, Y. Altered object-in-place recognition memory, prepulse inhibition, and locomotor activity in the offspring of rats exposed to a viral mimetic during pregnancy. Neuroscience 201, 184-198 (2012).

53. Szatmari, P. et al. Sex differences in repetitive stereotyped behaviors in autism: implications for genetic liability. Am. J. Med. Genet. B Neuropsychiatr. Genet. 159B, 5-12 (2012)

54. Mandy, W. et al. Sex differences in autism spectrum disorder: evidence from a large sample of children and adolescents. J. Autism Dev. Disord. 42, 1304-1313 (2012).

55. Mac Giollabhui, N. et al. Maternal inflammation during pregnancy and offspring psychiatric symptoms in childhood: timing and sex matter. J. Psychiatr. Res. 111, 96-103 (2019).

56. Rana, S. A., Aavani, T. \& Pittman, Q. J. Sex effects on neurodevelopmental outcomes of innate immune activation during prenatal and neonatal life. Horm. Behav. 62, 228-236 (2012).

57. Braun, A. E. et al. "Females are not just 'protected' males": sex-specific vulnerabilities in placenta and brain after prenatal immune disruption. eNeuro 6 ENEURO.0358-0319 (2019). 
58. Onore, C. E., Nordahl, C. W., Van de Water, J. A., Rogers, S. J. \& Ashwood, P. Levels of soluble platelet endothelial cell adhesion molecule-1 and P-selectin are decreased in children with autism spectrum disorder. Biol. Psychiatry 72, 1020-1025 (2012)

59. Nordahl, C. W. et al. Brain enlargement is associated with regression in preschool-age boys with autism spectrum disorders. Proc. Natl Acad. Sci. USA 108, 20195-20200 (2011).

60. Nordahl, C. W. et al. High psychopathology subgroup in young children with autism: associations with biological sex and amygdala volume. J. Am. Acad. Child Adolesc. Psychiatry S0890-8567, 30018-30016 (2020).

61. Lord, C. et al. Diagnosing autism: analyses of data from the Autism Diagnostic Interview. J. Autism Dev. Disord. 27, 501-517 (1997).

62. Lord, C. et al. The autism diagnostic observation schedule-generic: a standard measure of social and communication deficits associated with the spectrum of autism. J. Autism Dev. Disord. 30, 205-223 (2000).

63. Hus, V., Gotham, K. \& Lord, C. Standardizing ADOS domain scores: separating severity of social affect and restricted and repetitive behaviors. J. Autism Dev. Disord. 44, 2400-2412 (2014)

64. Gotham, K., Pickles, A. \& Lord, C. Standardizing ADOS scores for a measure of severity in autism spectrum disorders. J. Autism Dev. Disord. 39, 693-705 (2009).

65. Achenbach, T. M. Manual for the Child Behavior Checklist/4-18 and 1991 Profile (Department of Psychiatry, University of Vermont, Burlington, VT, 1991).

66. Pandolfi, V., Magyar, C. I. \& Dill, C. A. Confirmatory factor analysis of the child behavior checklist 1.5-5 in a sample of children with autism spectrum disorders. J. Autism Dev. Disord. 39, 986-995 (2009).

67. Schmeck, K et al. Discriminant validity of the child behaviour checklist CBCL-4/ 18 in German samples. Eur. Child Adolesc. Psychiatry 10, 240-247 (2001).

68. Brown, A. S. \& Meyer, U. Maternal immune activation and neuropsychiatric illness: a translational research perspective. Am. J. Psychiatry 175, 1073-1083 (2018).

69. Basil, P. L. et al. Prenatal maternal immune activation causes epigenetic differences in adolescent mouse brain. Transl. Psychiatry 4, e434 (2014).

70. Lombardo, M. V. et al. Maternal immune activation dysregulation of the fetal brain transcriptome and relevance to the pathophysiology of autism spectrum disorder. Mol. Psychiatry 23, 1001-1013 (2018).

71. Frick, L. R., Williams, K. \& Pittenger, C. Microglial dysregulation in psychiatric disease. Clin. Dev. Immunol. 2013, 608654 (2013).
72. Salter, M. W. \& Stevens, B. Microglia emerge as central players in brain disease Nat. Med. 23, 1018-1027 (2017).

73. Prins, J. R., Eskandar, S., Eggen, B. J. L. \& Scherjon, S. A. Microglia, the missing link in maternal immune activation and fetal neurodevelopment; and a possible link in preeclampsia and disturbed neurodevelopment? J. Reprod. Immunol. 126, 18-22 (2018).

74. Vogel Ciernia, A., Careaga, M., LaSalle, J. M. \& Ashwood, P. Microglia from offspring of dams with allergic asthma exhibit epigenomic alterations in genes dysregulated in autism. Glia 66, 505-521 (2018).

75. Koyama, R. \& Ikegaya, Y. Microglia in the pathogenesis of autism spectrum disorders. Neurosci. Res. 100, 1-5 (2015).

76. Takano, T. Role of microglia in autism: recent advances. Dev. Neurosci. 37, 195-202 (2015)

77. Abrahamsson, T. R. et al. A Th1/Th2-associated chemokine imbalance during infancy in children developing eczema, wheeze and sensitization. Clin. Exp. Allergy 41, 1729-1739 (2011).

78. Darlenski, R., Kazandjieva, J., Hristakieva, E. \& Fluhr, J. W. Atopic dermatitis as a systemic disease. Clin. Dermatol 32, 409-413 (2014).

79. Vandenbulcke, L., Bachert, C., Van Cauwenberge, P. \& Claeys, S. The innate immune system and its role in allergic disorders. Int. Arch. Allergy Immunol. 139, 159-165 (2006).

80. Schmatz, M., Madan, J., Marino, T. \& Davis, J. Maternal obesity: the interplay between inflammation, mother and fetus. J. Perinatol. 30 441-446 (2010).

81. Hantsoo, L., Kornfield, S., Anguera, M. C. \& Epperson, C. N. Inflammation: a proposed intermediary between maternal stress and offspring neuropsychiatric risk. Biol. Psychiatry 85, 97-106 (2019).

82. Rahman, T. et al. Effects of immune activation during early or late gestation on $\mathrm{N}$-methyl-d-aspartate receptor measures in adult rat offspring. Front. Psychiatry 8, 77 (2017).

83. Meyer, $U$. et al. The time of prenatal immune challenge determines the specificity of inflammation-mediated brain and behavioral pathology. J. Neurosci. 26, 4752-4762 (2006).

84. Shi, Y. H. et al. Coexistence of Th1/Th2 and Th17/Treg imbalances in patients with allergic asthma. Chin. Med. J. (Engl.) 124, 1951-1956 (2011).

85. O'Garra, A. \& Robinson, D. Development and function of T helper 1 cells. Adv. Immunol. 83, 133-162 (2004). 